This item was submitted to Loughborough's Research Repository by the author.

Items in Figshare are protected by copyright, with all rights reserved, unless otherwise indicated.

\title{
Generation of oblique dark solitons in supersonic flow of Bose-Einstein condensate past an obstacle
}

PLEASE CITE THE PUBLISHED VERSION

\section{LICENCE}

CC BY-NC-ND 4.0

\section{REPOSITORY RECORD}

El, G.A., A. Gammal, and A.M. Kamchatnov. 2019. "Generation of Oblique Dark Solitons in Supersonic Flow of Bose-einstein Condensate Past an Obstacle”. figshare. https://hdl.handle.net/2134/2463. 


\section{Loughborough University}

Generation of oblique dark solitons in supersonic flow of Bose-Einstein condensate past an obstacle

G.A. $\mathrm{El}^{\mathrm{a}}$, A. Gammal ${ }^{\mathrm{b}}$, and A.M. Kamchatnov ${ }^{\mathrm{c}}$

${ }^{a}$ Department of Mathematical Sciences, Loughborough University, Loughborough LE11 3TU, UK

bInstituto de Física, Universidade de São Paulo, 05315-970, C.P.66318 São Paulo, Brazil

'Institute of Spectroscopy, Russian Academy of Sciences, Troitsk, Moscow Region, 142190, Russia

\section{Introduction}

Nonlinear and dispersive properties of Bose-Einstein condensate (BEC) provide a possibility of formation of various nonlinear structures such as vortices and bright and dark solitons (see, e.g., [1]). Yet another type of nonlinear wave patterns has been observed in a series of experiments on the BEC flow past macroscopic obstacles [2]. In [3] these structures have been associated with spatial dispersive shock waves. Spatial dispersive shock waves represent dispersive analogs of the the well-known viscous spatial shocks (oblique jumps of compression) occurring in supersonic flows of compressible fluids past obstacles. In a viscous fluid, the shock can be represented as a narrow region within which strong dissipation processes take place and the thermodynamic parameters of the flow undergo sharp change. On the contrary, if viscosity is negligibly small compared with dispersion effects, the shock discontinuity resolves into an expanding in space oscillatory structure which transforms gradually, as the distance from the obstacle increases, into a "fan" of stationary solitons. If the obstacle is small enough, then such a "fan" reduces to a single spatial dark soliton [4]. Here we shall present the theory of these new structures in BEC.

\section{Generation of spatial dark solitons in an expanding BEC}

In the mean-field approximation, dynamics of BEC is described very well by the GrossPitaevskii (GP) equation [1]

$$
i \frac{\partial \psi}{\partial t}=-\frac{1}{2} \Delta \psi+V(\mathbf{r}) \psi+|\psi|^{2} \psi
$$

which is written in standard non-dimensional units (see, e.g., [3]). Here $\psi(\mathbf{r})$ denotes the condensate order parameter and $V(\mathbf{r})$ is the potential of the external forces acting on the condensate, as, e.g., the confining potential of the trap and/or the potential of an obstacle inside the BEC. In [2], the flow arising due to free expansion of a BEC after its release from the trap was actually two-dimensional, $\mathbf{r}=(x, y)$, and radially symmetrical. To illustrate 


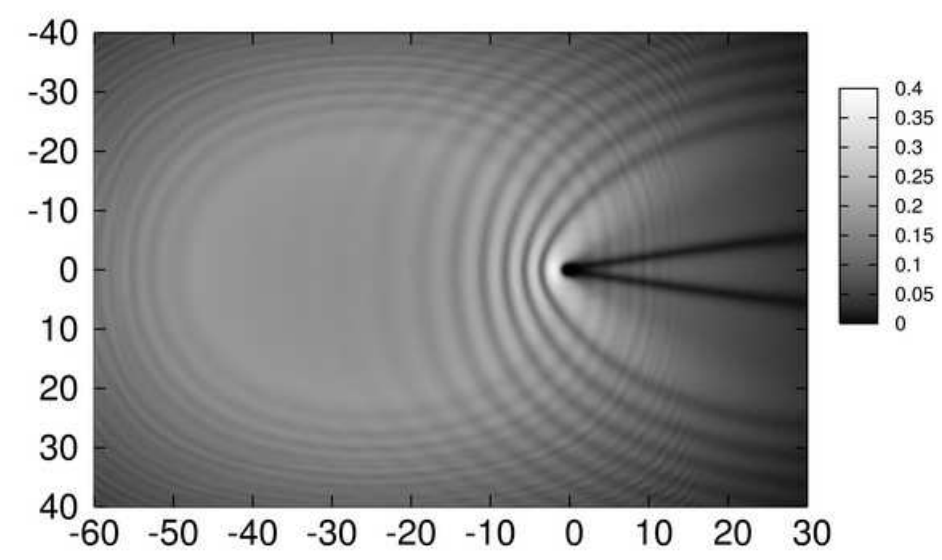

Figure 1. Generation of a pair of spatial dark solitons by an impenetrable disc of radius $r=1$ placed at $(0,0)$ in a BEC radially expanding from the center at $(-25,0)$. The density plot is shown at $t=12$. Initial radius of the condensate $R=25$.

the process of generation of waves by the obstacle, we have studied the time-dependent numerical solution of the GP equation (1) where $V(\mathbf{r})$ corresponds to interaction of the obstacle with the condensate. In our simulations, the flow was modeled by a free expansion of a BEC after its release from strongly confining trap, and the obstacle was modeled by an impenetrable disk with radius $r=1$. The results of these simulations are shown in Fig. 1 where a pair of spatial dark solitons is clearly seen behind the obstacle. It is clear that radial symmetry is not essential for a phenomenon of generation of such solitons. In fact, in real experiments the flow near the obstacle can be approximated by a uniform flow with constant velocity $M$ where in our notation $M$ is the Mach number. The conditions for good enough accuracy of such an approximation can be found as follows.

It is convenient to temporarily return to dimensional units. To be definite, we shall consider here free expansion of a BEC in a pancake geometry, that is the condensate is supposed to be confined in axial direction by a strong potential, so that its axial size is given by $a_{\|}=\sqrt{\hbar / m \omega_{\|}}, \omega_{\|}$being the frequency of the harmonic potential in the axial direction. Let the transverse frequency $\omega_{\perp}$ be small enough so that the Thomas-Fermi approximation is applied to the distribution of BEC in radial (transverse) direction. Then, before switching off the transverse potential the stationary radial density distribution is given by

$$
n(r)=C\left(1-r^{2} / l^{2}\right), \quad C=m \omega_{\perp}^{2} l^{2} / 2 g_{2 D},
$$

where $l=\left((16 / \sqrt{2 \pi}) N a_{\|}^{3} a_{s} \omega_{\|}^{2} / \omega_{\perp}^{2}\right)^{1 / 4}$ is the radius of BEC before its release from the trap, and $g_{2 D}=g / \sqrt{2 \pi} a_{\|}$is the effective nonlinearity constant in the pancake geometry.

An explicit time-dependent solution of the cylindrical GP equation for the condensate freely expanding in radial direction from the initial equilibrium state (2) is given by (see, 
e.g., $[5])$

$$
n(r, t)=\frac{C}{1+\omega_{\perp}^{2} t^{2}}\left[1-\frac{r^{2}}{l^{2}\left(1+\omega_{\perp}^{2} t^{2}\right)}\right], \quad u(r, t)=\frac{\omega_{\perp}^{2} t r}{1+\omega_{\perp}^{2} t^{2}} .
$$

Now, let us consider the regions of BEC not too far from the axis, $r \ll l \omega t$, for $t \gg \omega_{\perp}^{-1}$. Then in these approximations we obtain

$$
n(r, t) \cong C / \omega^{2} t^{2}, \quad u(r, t) \cong r / t
$$

that is the density is practically uniform but varying with time. In the same approximation the local healing length $\xi$ and the local sound velocity $c_{s}$ are given by $\xi=\hbar t / m l, \quad c_{s}=$ $l / \sqrt{2} t$. Hence, the local Mach number $M=u / c_{s} \cong \sqrt{2} r / l$ asymptotically does not depend on $t$. Thus, we get a supersonic flow past obstacle if we place the obstacle at the distance $d>l / \sqrt{2}$ from the axis.

Now, the flow can be considered as uniform if the size of the obstacle (scaled here as the healing length $\xi$ for a chosen moment of observation) satisfies the condition $\xi / d \ll 1$. For $d \sim l$ this gives

$$
t \ll m l^{2} / \hbar \text {. }
$$

This condition does not contradict to the condition $t \gg 1 / \omega_{\perp}$ if $N a_{s} / a_{\|} \gg 1$ which is nothing but the standard condition of applicability of the Thomas-Fermi approximation supposed in the beginning of our discussion.

At last, the characteristic time of establishing the soliton distribution is about $t \sim$ $\xi / c_{s} \sim m \xi^{2} / \hbar$ and also satisfies the condition (5), so that the flow can be considered as quasi-stationary.

Thus, we have shown for this example of BEC dynamics that the conditions of homogeneity, uniformity and quasi-stationarity of the flow can be satisfied in experiments with BEC. Thus, we can simplify our treatment of oblique dark solitons by supposing that the flow is uniform and homogeneous, $\mathbf{u}=(M, 0)$ at $|x| \rightarrow \infty$, where $M$ denotes the ratio of the asymptotic velocity of the flow to the sound velocity corresponding to the asymptotic value of the density $n=n_{0}=1$. Let at the initial moment $t=0$ there be no disturbance in the condensate so that it is described by the plane wave function

$$
\left.\psi(x, y)\right|_{t=0}=\exp (i M x)
$$

corresponding to a uniform condensate flow. To be specific, let us take $M=5$. The density plot at $t=20$ is shown in Fig. 2. It can be clearly seen how a pair of oblique solitons is formed behind the obstacle. As our simulations show, their length grows with time and, except in the vicinity of the end points, the density distributions does not demonstrate any vorticity.

\section{Oblique soliton: analytic solution}

To obtain an analytic description of spatial dark solitons in a BEC, we notice that far enough from the obstacle its potential can be neglected, so we transform Eq. (1) to the 

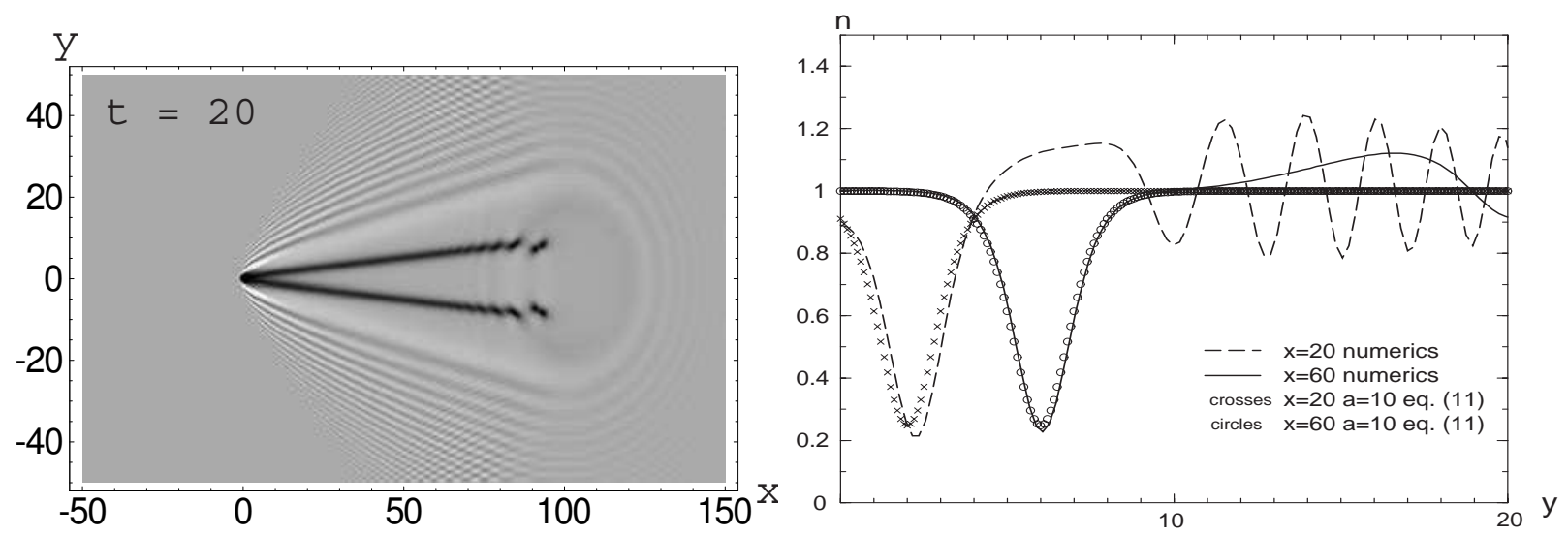

Figure 2. Generation of a pair of oblique dark Figure 3. Cross sections of the density dissolitons after "switching on" a uniform su- tributions for $x=20$ (dashed line), $x=60$ personic flow $(M=5)$ past a disk-shaped im- (solid line) and $y>0$ obtained from numerpenetrable obstacle of radius $r=1$ located at ical solution of the GP equation $(1)(M=5$, $(0,0)$. The direction of the flow is from left to $t=20)$. These are compared with soliton right. Density plot is shown for $t=20$. The profiles (13) with slope $a=10$ shown as funcdark structures correspond to oblique dark tions of $y$ at the same values of $x(x=20$ solitons, which in turn generate the vortex corresponds to "crosses" and $x=60$ to "cirstreets near the end points. cles").

so-called "hydrodynamic form" by substitution

$$
\psi(\mathbf{r})=\sqrt{n(\mathbf{r})} \exp \left(i \int^{\mathbf{r}} \mathbf{u}\left(\mathbf{r}^{\prime}\right) d \mathbf{r}^{\prime}\right) \exp (-i \mu t)
$$

Substituting (7) into (1) and separating real and imaginary parts we obtain a system of equations for the density $n(x, y)$ and the velocity field $\mathbf{u}=(u(x, y), v(x, y))$,

$$
\begin{array}{r}
(n u)_{x}+(n v)_{y}=0, \\
u u_{x}+v u_{y}+n_{x}+\left(\frac{n_{x}^{2}+n_{y}^{2}}{8 n^{2}}-\frac{n_{x x}+n_{y y}}{4 n}\right)_{x}=0 \\
u v_{x}+v v_{y}+n_{y}+\left(\frac{n_{x}^{2}+n_{y}^{2}}{8 n^{2}}-\frac{n_{x x}+n_{y y}}{4 n}\right)_{y}=0
\end{array}
$$

where we have omitted tildes for convenience. Our aim now is to find the solution of this system under the conditions that the BEC flow is uniform at infinity

$$
n=1, \quad u=M, \quad v=0 \text { at }|x| \rightarrow \infty,
$$

where $M$ denotes the ratio of the asymptotic velocity of the flow to the sound velocity, i.e. the Mach number.

Let us seek a solution of the form $n=n(\theta), u=u(\theta), v=v(\theta)$, where $\theta=x-a y$ and $a$ denotes the slope of a line with a given phase (e.g., location of a wave crest) with the 
$y$-axis. Substitution of this ansatz into the system (8) followed by a simple integration, yields expressions for the velocity components in terms of the density

$$
u=\frac{M\left(1+a^{2} n\right)}{\left(1+a^{2}\right) n}, \quad v=-\frac{a M(1-n)}{\left(1+a^{2}\right) n},
$$

where the integration constants were chosen according to condition (9). Besides that, we get the ordinary differential equation for the density

$$
\frac{1}{4}\left(1+a^{2}\right)\left(n_{\theta}^{2}-2 n n_{\theta \theta}\right)+2 n^{3}-(2+p) n^{2}+p=0,
$$

where

$$
p=M^{2} /\left(1+a^{2}\right) .
$$

Equation (11) with the condition (9) for the density at infinity can be easily integrated (see [4] for details) to give the desired solution in the form of a dark soliton

$$
n(\theta)=1-\frac{1-p}{\cosh ^{2}\left[\sqrt{1-p} \theta / \sqrt{1+a^{2}}\right]} .
$$

The velocity components can then be found by substitution of this solution into Eqs. (10). Thus, formula (13) gives the exact dark spatial soliton solution of the GP equation. We call it "oblique" because it is always inclined with respect to the direction of the supersonic flow. To compare the analytic solution (13) with the results of numerical simulations, we have calculated the parameter $p$ from Eq. (12) using the value of the slope $a$ inferred from the numerical solution. A comparison of the theoretical profile of the oblique dark soliton given by Eq. (13) with the corresponding part of the density profile in the full numerical solution is shown in Fig. 3. The excellent agreement between these two profiles confirms that the line patterns in Fig. 2 are indeed oblique dark solitons generated by the obstacle.

The developed here theory explains qualitatively the patterns observed in the experiment [2] where the large amplitude nonlinear waves generated by the BEC flow past an obstacle are clearly seen.

AMK thanks EPSRC and RFBR (grant 05-02-17351) and AG thanks FAPESP and $\mathrm{CNPq}$ for financial support.

\section{REFERENCES}

1. L.P. Pitaevskii and S. Stringari, Bose-Einstein Condensation, Cambridge University Press, Cambridge, 2003.

2. E.A. Cornell, talk at the Conference on Nonlinear Waves, Integrable Systems and their Applications (Colorado Springs, June 2005); available at http://jilawww.colorado.edu/bec/papers.html.

3. G.A. El and A.M. Kamchatnov, Phys. Lett A 350 (2006) 192 ; erratum: Phys. Lett. A 352 (2006) 554 .

4. G.A. El, A. Gammal and A.M. Kamchatnov, Oblique dark solitons in supersonic flow of a Bose-Einstein condensate, Phys. Rev. Lett. (2006) (to appear); also e-print nlin.PS/0604044.

5. A.M. Kamchatnov, JETP 94 (2004) 908. 